\title{
Et le prix, c'est pour... \\ pour une approche anthropologique \\ des festivals et des genres musicaux.
}

\author{
Fernanda Marcon \\ Université Fédérale de Santa Catarina
}

\section{Introduction}

Je me suis toujours intéressée - comme anthropologue, mais aussi comme spectatrice et participante - aux festivals de musique. C'est la raison pour laquelle j'ai réalisé une ethnographie sur les processus qui président à la composition des « musiques de festival " (Marcon 2009) ${ }^{1}$. Dans ce travail, j’ai observé comment la dynamique des festivals déterminait une situation particulière en ce qui concerne, d'une part, la composition - conditionnée par l'appréciation du jury, le coût en argent et en temps passé (le temps d'exécution), caractérisée par le rapport avec le public, la scène et l'interdiction du droit à l'erreur - et, d'autre part, l'appréciation/plaisir - c'est à dire surtout le sentiment de faire de la musique ensemble et de faire ensemble une musique qui remporte un prix. De plus, je me suis intéressée à la recherche concernant l'émergence et la formation des genres musicaux joués dans ces événements, en d'autres termes, l'influence des contextes d'exécution sur la caractérisation des genres musicaux. Ainsi, nous proposons d'observer les genres musicaux à partir de leur dynamique constituante, en ayant recours à la notion des genres de discours de Bahktin (1997) pour comprendre comment les genres musicaux se constituent de façon fluide dans les différentes sphères de communication, parmi lesquelles les festivals de musique sont des cas paradigmatiques.

1 Je fais référence à mon mémoire de master, soutenu en juillet 2009 dans le Programme d'Études supérieures en Anthropologie sociale de l'Université Fédérale de Santa Catarina, intitulé "Musique de Festival: une ethnographie de la production de musique nativiste dans le festival Sapecada da Canção Nativa à Lages-SC-BR" ("Música de Festival: uma etnografia da produção de música nativista no festival Sapecada da Canção Nativa em Lages-SC”). 
La construction relationnelle des festivals et des genres musicaux sera étudiée à partir de deux modèles de festivals brésiliens : les festivals de MPB des années 1960 et les festivals de musique nativiste - apparus dans les années 1970 et qui dont on trouve encore des exemples dans le sud du Brésil. Le choix de ces deux modèles de festivals est dû à l'intérêt d'une approche comparative qui prend en compte le processus de formation des genres musicaux - dans ce cas, la MPB et la musique nativiste - à partir des festivals et de l'importance de la réflexion anthropologique sur le concept même de genre musical et sur l'incidence de cette réflexion sur la production de connaissance musicale.

\section{Les Festivals de Chanson: populaire et native.}

La musique qui est aujourd'hui connue sous le nom de MPB (la musique populaire brésilienne synthétisée dans un sigle avec des majuscules) après les années 1960, montre un rapport intéressant avec les festivals. D’après Napolitano (2007), les années 1960 ont été marquées par un changement radical dans le panorama phonographique brésilien. À côté de l'émergence de la télévision, qui s'établit bientôt comme véhicule de masse de la musique, le marché musical brésilien se lance dans la " substitution des importations ", selon Napolitano, ce qui déclenche une augmentation considérable de la consommation de chansons composées, interprétées et produites dans le pays (Napolitano 2007: 89).

La confluence d'une période de fortification politique et économique et d'un marché culturel en développement crée un contexte idéal que mettent en évidence les récits sur l'origine de la bossa-nova comme genre musical capable de représenter la musique brésilienne moderne². D’après Napolitano, la bossa-nova devient mondialement connue à partir de 1959/1960, après l'arrivée de l'album Chega de Saudade, du compositeur João Gilberto³. La

\footnotetext{
2 Selon Napolitano et Wasserman (200o), en ce qui concerne le problème de son origine, l'historiographie de la musique populaire brésilienne présente deux branches principales : la première est la recherche de la souche authentique de la musique populaire brésilienne. La deuxième - celle des auteurs - cherche à susciter une réflexion historiographique qui met l'accent sur la pluralité des vecteurs qui constituent la musique populaire brésilienne et, au même temps, remettent en question l'importance de l'enquête sur les origines (Napolitano et Wasserman 2000: 168).

3 Toutefois, comme l'a démontré le Professeur Allan de Paula Oliveira dans sa conférence au Département d'anthropologie de l'Université Fédérale de Santa Catarina, en septembre 2010,
} 
bossa-nova passe alors des cercles privés - les appartements et les boîtes de la zone sud de Rio de Janeiro - aux universités et aux théâtres de São Paulo.

La période qui précède le premier festival de MPB offre un panorama encore plus intéressant de ce que recouvre ce sigle dans le contexte des festivals : la relation complexe entre des musiciens de bossa-nova nationalistes - qui veulent dissoudre l'élitisme du genre en se rapprochant de la tradition des sambas anciennes ${ }^{4}$ - et les intellectuels des Centres populaires de culture (CPCs) de l'Union nationale des étudiants (UNE)s.

Cette relation devient complexe du fait que, malgré le lien de certains compositeurs (comme Geraldo Vandré) avec les mouvements politiques des étudiants, la plupart des compositeurs politiquement engagés de bossa-nova n'aimait pas les prémisses esthétiques et idéologiques du Manifeste - rédigé par l'économiste Carlos Estevam Martins - que le Centre populaire de culture de l'UNE a publié en 1962. D'après ce document, la chanson engagée devait soumettre la forme au contenu (nationaliste) et l'expression artistique à la communication du " peuple».

Après 1965, motivée par le bon accueil qui a été fait au festival de San Remo, en Italie, la chaîne de télévision Excelsior, de São Paulo, organise le « Premier Festival national de musique populaire brésilienne ». Dans ce festival, la chanson "Arrastão ", composée par Edu Lobo et Vinícius de Moraes et interprétée par Élis Regina, reçoit le premier prix. D’après un entretien avec le producteur musical Solano Ribeiro - qui fut l'instigateur de ce

l'historiographie des genres de musique populaire brésilienne surestime l'idée de la rupture, c'est-àdire, les éléments innovants que les genres musicaux, comme la bossa-nova, ont introduit sur la scène musicale brésilienne. Dans cette perspective, la rupture est parfois vue comme un facteur positif, d'autres fois, comme un facteur négatif, ce qui amène Oliveira à comparer les discours d'Augusto de Campos et de José Ramos Tinhorão sur l'émergence de la bossa-nova.

4 En 1961, l'arrivée sur le marché du disque de la chanson “Quem quiser encontrar o amor", de Carlos Lyra et Geraldo Vandré vient consolider une nouvelle branche de la bossa-nova : la bossa-nova « nationaliste " caractérisée par son message, " plus politisé " (Napolitano 2007: 70-72).

5 D’après Renato Ortiz (2005), le Centre populaire de culture a fonctionné de 1962 à 1964 en partenariat avec l'Union nationale des étudiants. En matière d'action politique, un des axes principaux de ce mouvement se caractérisait par la réflexion menée sur le rôle que jouaient les intellectuels dans l'organisation de la culture. Selon cette branche, fortement inspirée par Gramsci, l'intellectuel devait faire " partie du peuple », devenir peuple. La catégorie d'aliénation, quand elle est appliquée aux arts finit par consacrer la culture populaire comme étant la manifestation authentique et vraie de la culture du peuple. Cette catégorisation des arts, restreinte au plan politique et penchant vers le nationalisme menait à une conception selon laquelle l'artiste devait être engagé et parler du peuple et pour le peuple, en prenant ses distances avec le plaisir esthétique susceptible de faire obstacle à son action politique (Ortiz 2005: 68-75). 
premier festival - sur le site « O Toque ", en 2006, le sigle MPB commença à être utilisé par les médias après le deuxième festival, pour abréger le nom de l'événement ${ }^{6}$. Les années suivantes, d'autres chaînes de télévision - comme la Record et la carioca Globo - investirent sur ce format d'émission et ont aussi commencé à promouvoir leurs propres festivals. Solano Ribeiro, de la télévision Record, fut ainsi l'organisateur du Deuxième festival de MPB, en 1966. Ce festival devint nationalement connu grâce à la dispute entre la chanson " Disparada ", de Geraldo Vandré et Theo de Barros, et "A Banda ", de Chico Buarque ${ }^{7}$. L'année suivante, toujours à la télévision Record, fut organisé le festival considéré comme étant le plus important de l'époque, celui de 1967, qui consolida définitivement ce genre d'émission à la télévision ${ }^{8}$.

C'est au public que se devait principalement la forte compétitivité qui régnait dans les festivals. C'est la raison pour laquelle le compositeur Sérgio Ricardo, dans le documentaire "Une nuit en 67 ", se déclare indigné qu' « un public soit d'un coup devenu un personnage ». Au cours du Festival international de la chanson (FIC) de 1968 - réalisé par la télévision Globo et organisé par Solano Ribeiro - le mécontentement de la jeunesse de gauche à l'égard des chansons qui ne ressemblaient pas à des chants de lutte contre la dictature - les chansons de protestation - apparut de manière encore plus évidente. Le compositeur Caetano Veloso, icône du mouvement tropicaliste ${ }^{9}$, se heurta à un public qui vibrait pour sa rivale « Caminhando ou Pra não dizer que não falei

6 Entretien disponible en: http://www.otoque.com.br/detalhes_entrevista_outras.asp?id_cat=1, consulté le 29 septembre 2010.

7 Les deux chansons finissent par partager le premier prix au festival.

8 En 2010 a été lancé le documentaire « Une nuit en 67 " ("Uma noite em 1967) ", réalisé par Renato Terra et Ricardo Calil, Zuza Homem de Mello étant consultant. Dans son blog sur l'internet, le 3 août 2010, le producteur musical Solano Ribeiro se montre mécontent de l'édition du documentaire. Selon Ribeiro, les réalisateurs n'ont pas respecté son objectif de diffuser la musique populaire brésilienne, ils ont mis l'accent sur les intérêts commerciaux de l'émission. L'information est disponible sur : http:/| solanoribeiro.blogspot.com/, consulté le 26 septembre 2010.

9 Le mouvement tropicaliste émerge des festivals de la chanson des années 1960 et se caractérise par l'influence des courants artistiques considérés comme d'avant-garde (comme la bossa-nova) et de la culture " pop " internationale. De plus, le mouvement était un projet culturel inséré dans les structures de l'industrie culturelle - comme le montre Napolitano. Cependant, le tropicalisme assimilait les critiques de la contre-culture au capitalisme et aux codes établis. Même si ses manifestations avaient lieu essentiellement sur la scène musicale - les principaux réprésentants en étant les musiciens Caetano Veloso, Gilberto Gil, Tom Zé, Maria Bethânia, Gal Costa et Torquatto Neto - le mouvement a été beaucoup diffusé dans d'autres sphères artistiques, comme le cinéma, le théâtre et les arts visuels. Le tropicalisme était fortement critiqué par les intellectuels de gauche pour son absence d'engagement politique - ce que ne niaient pas les tropicalistes. 
das flores ", de Geraldo Vandré ${ }^{10}$. Ce public hua beaucoup Chico Buarque et Tom Jobim, les compositeurs de "Sabiá ", la chanson lauréate.

Tous ces événements ont formé une " histoire des festivals de la chanson " qui, à son tour, a permis l'émergence de narrations spécifiques portant sur ce qui, aujourd'hui, est compris comme étant la MPB. Ainsi, l'éthique et l'esthétique des festivals finissent par définir une bonne partie du répertoire considéré, aujourd'hui, comme populaire, brésilien (ou national) et avancé"1 dans notre musique. Même si le sigle MPB réunit différents genres musicaux, ce qu'il importe de montrer c'est que la MPB est devenue un genre musical en soi grâce aux festivals de la chanson. Avant ces événements, la MPB n'existait pas comme classification commerciale dans les maisons de disques. De plus, avec le sigle, on inaugure un moyen nouveau et efficace de production musicale : plusieurs musiciens commencent à composer et jouer ce qu'ils appellent la MPB. Par contre, d'autres genres musicaux continuent à exister (ou commencent à exister) au Brésil, sans, pour autant, être englobés par ce sigle. Qu'est-ce que cette sélectivité veut dire ? Autant les critères qui déterminent le marché et l'industrie phonographiques, que les nouvelles acceptions des catégories populaire et brésilienne dans la production musicale, peuvent aider à répondre à cette question. Cependant, on ne peut pas perdre de vue - notamment dans une approche anthropologique - (la façon dont le concept de genre musical instaure des rapports qui ont une incidence sur la production

10 Dans un entretien récent pour le réseau de télévision GloboNews, le compositeur Geraldo Vandré a nié avoir composé des chansons de protestation et s'être engagé sur des positions politiques antimilitaristes à l'époque des festivals. Évidemment, les témoignages de Vandré, après sa période d'expatriation, sont sujets à controverse. Beaucoup croient que le musicien a été obligé de nier son activité militante. L'entretien donné au journaliste Geneton Moraes Neto le 25 septembre 2010 peut être suivi sur le site : http://historica.me/video/geraldo-vandre-quebra-o\#ixzz1of7glBFW, consulté le 27 septembre 2010.

11 D'après Menezes Bastos (2009), la sélection opérée par le sigle MPB - qui exclut plusieurs genres de son répertoire de chansons - révèle une perception selon laquelle la musique populaire brésilienne est liée au " développement " politique, moral, esthétique et musical (Menezes Bastos 2009: 3). Dans un autre article, Menezes Bastos (1999) montre que l'étude de la musique en Amérique Latine, surtout des genres de la musique populaire, doit être attentivement évaluée en ce qui concerne sa relation paradigmatique avec l'émergence de l'axe jazz-rock en Occident, principalement pendant sa consolidation dans les années 1930-196o. D’après l'auteur, la musique populaire représente une manifestation globale de la modernité récente, car elle s'insère dans un discours de " déterritorialisation " dans lequel la phonographie, la radio et le cinéma sont fondamentaux. Pour Menezes Bastos, le XXe siècle a été marqué par un processus de redéfinition de ce qui était considéré comme " musique nationale " et comme " populaire ", sans avoir en tête l'idée d'une autoélaboration re-signifiante. La musique populaire brésilienne des années 1960, en se constituant dans l'univers d'un discours en même temps modernisateur et de forte répression politique, a pu diffuser - à travers des festivals de musique et à la télévision surtout - une nouvelle perception du Brésil, une nouvelle façon d'écouter le Brésil. 
de connaissance en matière musicale et sur la manière dont les musiques sont différenciées, classées et décrites.

À la suite de l'instauration, en 1968, de l'Acte institutionnel n ${ }^{\circ} 5$ (AI-5) ${ }^{12}$ la plupart des festivals de MPB entrent en déclin (après 1970). Toutefois, dans le sud du pays, on a un mouvement contraire : les festivals de chanson se développent, c'est le début de l'âge des festivals nativistes - en paraphrasant le titre du livre de Zuza Homem de Mello (2003), L'âge des festivals: une parabole.

C'est à travers ces festivals que la musique nativiste se constitue comme genre musical du sud du Brésil à partir des années 1970. Ces événements commencent à faire partie du calendrier des fêtes de plusieurs villes du Rio Grande do Sul et, après, des États de Santa Catarina, Paraná et Mato Grosso do Sul. Ce sont des concours de musique, où sont octroyés des prix en argent et des trophées, avec à la clé l'enregistrement des chansons finalistes. La musique nativiste, comme la MPB, finit par englober plusieurs genres musicaux comme la vaneira (ou le vaneirão), la milonga et la chamarra - la plupart définie par des chercheurs et des folkloristes, comme Barbosa Lessa et Paixão Cortes, comme des genres « traditionnels » du Rio Grande do Sul. Le terme " traditionnel » est, ici, lié à l’avènement des Centres de tradition gaucha (les « CTG ») - créés, en 1948, par les chercheurs que nous venons de mentionner - qui sont à l'origine de l'institutionnalisation et de la centralisation des CTG et de leurs activités ${ }^{13}$. Après les années 1970, quelques musiciens du Rio Grande do Sul commencent à remettre en question les règles des manuels des CTG, principalement en ce qui concerne l'interdiction de certains instruments musicaux

12 L'Acte institutionnel $n^{\circ} 5$ (AI-5) a été promulgué par les militaires après le coup d'État de 1964. L'AI-5 donnait un pouvoir extraordinaire au Président de la République. Il suspendait plusieurs garanties institutionnelles. La censure devient encore plus forte et crée énormément d'obstacles à la réalisation des festivals de la chanson.

13 Les CTG et le MTG sont considérés comme faisant partie de la vaste gamme de mouvements régionalistes du sud du Brésil. Ils sont responsables d'un projet de revitalisation de la culture gaucha. Lucas identifie trois mouvements différents dans le cadre de ces mouvements de revitalisation : le premier, à la fin du XIXème siècle, caractérisé par l'idéalisation, par les intellectuels locaux, des clubs de loisirs pour cultiver des traditions gauchas. Le deuxième, après la deuxième Guerre Mondiale, est caractérisé par la création des Centres de tradition gaucha (CTG) par des étudiants des écoles secondaires de Porto Alegre et par l'institutionnalisation du Mouvement traditionaliste gaucho (MTG). Enfin, le troisième mouvement est caractérisé par l'avènement, dans les années 1970, de ce qu'on appelle « nativisme ", dans le cadre des festivals de musique. D’après Oliven (1992), le Mouvement traditionaliste gaucho (MTG), fondé en 1966, en s'affirmant comme « le plus grand mouvement de culture populaire du monde occidental " veut que se construise une culture gaucha, premier pas vers la définition d'une culture brésilienne. Selon les intellectuels de ce mouvement, " on n'arrive au national qu’à travers le régional " (Oliven 1992: 128). 
(par exemple, les guitares électriques et la batterie) et de quelques genres perçus comme étant non traditionnels et venus des régions des frontières (comme le chamamé, la zamba et la chacarera). Au même temps, ces musiciens accusent la musique régionale d'être de mauvaise qualité et soutenue par des intérêts de marketing et cherchent à se différencier de la production musicale de compositeurs comme Pedro Raymundo, Zé Mendes, Teixeirinha et d'autres groupes musicaux qui jouaient et qui jouent encore aujourd'hui dans des bals gauchos, dans tout le sud du Brésil. Pedro Raymundo (né dans le Santa Catarina en 1906 et mort en 1973) joue alors un rôle sans précédent. D’après Mann (2002), quand Raymundo arrive à Porto Alegre, en 1929, c'est déjà un musicien chevronné. « Il jouait du choro, des valses et même du jazz avec son accordéon, quand il luttait comme il le pouvait pour survivre " (Mann 2002: 14). Il explore les genres musicaux gauchos, il voyage dans la province du Rio Grande do Sul afin d'élaborer un style vestimentaire avant même que soient fixés les costumes qui caractérisent le mouvement traditionaliste. En 1939, il crée le "Quatuor des Tauras " et donne des concerts sur les radios Gaucha et Farroupilha, qui l'emploient pour qu'il fasse sa propre émission. Avec le Quatuor, Pedro Raymundo compose à profusion, il fait des adaptations des chansons du folklore gaucho - à l'exemple de Boi Barroso et de Prenda Minha - et part pour une tournée dans la province du Rio Grande, dans le Santa Catarina et dans le Paraná. D’après Mann, à la fin de cette tournée, il essaie de convaincre le groupe de le suivre à Rio de Janeiro. Il ne réussit pas et quitte le Quatuor pour partir tout seul. Sa réussite est très rapide. En 1943, après avoir patienté dans de nombreuses queues pour participer à des concours de chanteurs amateurs dans les radios de Rio, il arrive à présenter sa chanson « Gaúcho Alegre » à la Radio Mairynk Veiga - qui aura une audience record:

[...] Il se présente avec un chapeau qui tombe sur son front, des bottes, des éperons, une ceinture porte-monnaie et des culottes faites par sa femme. La chanson " Gaucho Alegre » lui rapporte la sympathie générale. Son personnage, qui allait bientôt devenir célèbre à l'échelle nationale - le " Gaucho gai de la Radio" - est alors constitué. Il est invité par la maison Continental à enregistrer un disque de 78 tours avec la chanson «Adeus, Mariana » (Mann 2002: 16).

Après l'énorme succès de "Adeus Mariana », Pedro Raymundo enregistrera, jusqu'à 1958, soixante disques de 78 tours, quarante d'entre eux chez Continental. Dans une interview au journal Pasquim, en 1971, Luiz Gonzaga 
affirme s'être inspiré de Pedro Raymundo pour créer son célèbre style vestimentaire:

Quand Pedro Raymundo est arrivé ici habillé en gaucho, je me suis senti nu. Je me suis demandé : pourquoi le nord-est n'a pas ses propres caractéristiques? J'ai pris la décision de créer quelque chose. Cela devrait être lié à Lampião. J'allais imiter cet homme, mais personne ne remarquerait que je le copie. Il était gaucho, j'allais être cangaceiro. (Luiz Gonzaga dans un entretien avec Ziraldo. Pasquim, $1971^{14}$ )

Le style de Pedro Raymundo a créé une lignée d'artistes gauchos comme Túlio Piva, Gildo de Freitas, Teixeirinha et Zé Mendes. Ces artistes parviennent à la scène nationale avec une production musicale que les écrivains 'nativistes', ensuite, ont déclaré être "commerciale » ou de "mauvaise qualité». Ainsi, la protection nationale - c'est-à-dire la commercialisation d'une musique qui était montrée du doigt comme étant un pastiche de la musique régionale gaucha - est devenue incompatible avec le projet nativiste qui voulait revitaliser le mouvement régional à travers une production musicale de bonne qualité. Selon Santi (2004), cette quête de qualité doit être comprise comme un effort pour suivre l'évolution de toute la musique brésilienne c'est l'exemple de la bossa-nova, du tropicalisme et de la chanson de protestation - ainsi que les transformations technologiques introduites dans le pays - comme les instruments musicaux électriques et amplifiés et l'évolution de la diffusion sonore.

D’après Lucas, les genres musicaux des festivals de musique nativiste, considérés comme traditionnels ou 'natifs' étaient, en fait, des réinterprétations locales des danses européennes importées aux Amériques au milieu du XIXème siècle. Dans le Rio Grande do Sul, ces danses remplacent et en même temps incorporent les danses importées par les colonisateurs des Açores et les Luso-Brésiliens du XVIIIème siècle (Lucas 1990: 211). D’après Oliveira et Verona (2006), à la fin du XIXème siècle, entrent en jeu des genres musicaux venus d'Argentine, d'Uruguay et du Paraguay. Cependant, au début, ces genres sont pratiqués par les compositeurs gauchos de façon timide et sont joués dans des bals, les fandangos ${ }^{15}$, en province - la milonga

14 D’après Mann (2002: 18).

15 Avec la création des premiers prix régionalistes, le fandango gaucho devient le représentant de ce 
et le chamamé en sont des exemples.

L'histoire du premier festival et du premier CTG établit comme un mythe de l'origine. Cette histoire met en exergue l'initiative courageuse du poète et compositeur Colmar Duarte. Elle est considérée comme absolument légitime par les traditionalistes, par les nativistes et aussi par la presse du Rio Grande do Sul. En 1970, une radio de la ville de Uruguaiana-RS commence à promouvoir un festival de musique populaire: le « Premier Festival de la Chanson populaire de la frontière ". D’après Lucas, la milonga intitulée « Abichornado $»^{16}$, composée par Colmar Duarte et Júlio Machado da Silva Filho, fut éliminée par le jury sous prétexte que c'était une « chanson gauchesque "( " música gauchesca "). Mécontent du résultat, Colmar Duarte décida alors de créer un festival de musique régionale. Toutefois, comme le fait remarquer Santi (2004), les motifs de l'élimination de cette chanson sont sujets à controverse : en effet, comme personne ne voulait chanter la chanson, les compositeurs avaient décidé de la chanter eux-mêmes (accompagnés par le groupe " Marupiaras »). Selon le jury, l'élimination était due aux caractéristiques trop régionales de la chanson. Cependant, Milton Mendes de Souza, qui, à l'époque, était présentateur à la radio, affirme que l'élimination était due au fait que l'interprète avait pleuré pendant l'exécution et n'était pas arrivé à terminer la chanson (Santi 2004: 54).

Quoi qu'il en soit, il est incontestable que ce festival a donné lieu à la création d'un événement du même format (un festival) destiné à diffuser la musique régionale du Rio Grande do Sul, un style de musique qui, selon Colmar Duarte, était la cible de préjugés. Pour concrétiser son but, le compositeur fit appel aux directeurs du CTG « Sinuelo do Pago », dans la ville d’Uruguaiana.

qui, plus tard, est connu comme étant les fondements de la musique folklorique du Rio Grande do Sul. Plusieurs chercheurs, comme Cezimbra Jacques (1979), Paixão Côrtes et Barbosa Lessa (1975) définissent le fandango comme un " bal " ou une " fête " réalisé dans les communautés rurales où on jouait différentes danses, décrites par Jacques comme étant des « danses de claquettes " ( danças sapateadas "). Ces danses sont accompagnées par une guitare de 10 ou 12 cordes (viola). D’après l'auteur, on les trouvait sous deux formes: l'une était faite pour être dansée et l'autre pouvait être chantée avec différents textes. Parmi les danses mentionnées par l'auteur on retrouve le cará, le xará, la serrana, l'anu, le tatu, la tirana et la chimarrita. La chimarrita, la tirana et l'anu perdurent jusqu'à la moitié du XIXème siècle dans les bals ruraux. Nous voulons ici non pas attester la véracité des données présentées par ces chercheurs et soutenir leur recherche quant à l'origine des genres, mais plutôt souligner la façon dont est ainsi présenté un classement particulier selon des catégorisations telles que " propagation européenne ", "propagation sud-américaine » et des genres propres au Rio Grande do Sul, notamment le classement employé par Oliveira et Verona.

16 D’après Santi, en argot gaucho le terme veut dire « triste ». (Santi 2004: 53) 
Cependant, il n'arriva pas à matérialiser son objectif. L'année suivante, est élu président de ce CTG, il fit de ce projet de festival une priorité. Le festival, idéalisé par Colmar Duarte, se concrétisa finalement en 1971, sous le nom de «Califórnia da Canção Nativa ». Sur la pochette de l'album du premier Califórnia on trouve l'explication du nom:

[...] vient du grec [et] veut dire 'ensemble de belles choses'. Dans le Rio Grande do Sul on appelle 'califórnias' les incursions que fait Chico Pedro dans la province de Cisplatina,, pour récupérer les biens brésiliens qui s’y sont enracinés et sur lesquels s'exercent des persécutions (1850). Plus tard, 'califórnia' désigne les courses de chevaux où concourent plus de deux animaux [...]. Mais le nom CALIFÓRNIA DA CANÇÃO NATIVA est choisi par ses inventeurs ${ }^{17}$ dans le sens $d$ '" ensemble de belles choses " et de " concours entre plusieurs personnes pour de grands prix ».

D’après un document dactylographié (intitulé «Histoire de la Califórnia da Cançao Nativa»), que nous avons retrouvé lors d'une visite à l'Institut de la tradition et du folklore gaucho (IGTF) à Porto Alegre, le premier Califórnia da Cançao Nativa eut lieu sur deux week-ends et 85 chansons furent alors enregistrées. Le premier week-end, du 8 au 10 décembre 1971, se déroulèrent les quarts de finale, au cours desquelles furent présentées douze chansons par soirée, six chansons étant sélectionnées chaque soir. Le deuxième week-end, du 17 au 18 du même mois, fut celui des «demi-finales ", au cours desquelles les dix-huit chansons choisies précédemment furent présentées. C'est parmi ces chansons que furent choisies les chansons finalistes qui figurent sur le disque. Ces chansons furent présentées une nouvelle fois lors de la finale, le 19 décembre 1971. Curieusement, la première chanson qui gagna le festival fut celle de Colmar Duarte et de Henrique Dias de Freitas Lima, une chanson triste (de " lamentation ») intitulée " Reflexão $»^{18}$.

Lors d'une interview donnée au journal « Folha da Tarde » de Porto Alegre, le 27 septembre 1971, Henrique Dias de Freitas Lima fit une critique

\footnotetext{
17 D’après Santi (2004: 55).

18 Cette composition a été classée par le jury du festival comme étant celle d'une chanson de « lamentation » en prenant en compte la forme musicale (qui équivaut à la catégorie analytique de genre, ou à l'idée de rythme, d'après nos interlocuteurs). En se référant au sommaire du jury, on peut constater que les chansons qui sont primées relèvent des genres suivants: « lamentation ", " chanson des missions» et " exaltation ». Parmi les chansons finalistes, on peut également retrouver les formes musicales: " acalanto ", " toada ", " chanson chote ", " chanson de la rivière » et " style ».
} 
féroce du " cap " que la musique gaucha était en train de prendre. Il considérait notamment qu'elle se caractérisait désormais par de " fausses paroles et mélodies, à l'exemple majeur de Teixeirinha ". Pour lui, "Coração de luto " de ce compositeur et « Pára Pedro » de Zé Mendes, par exemple " n’ont jamais été des thèmes gauchos ${ }^{19}$. Il est évident que par cette critique, il cherchait à démontrer l'importance du festival Califórnia en mettant l'accent sur la rupture ainsi opérée avec le passé, considéré, en l'occurrence, comme le principal responsable de la non-reconnaissance de la musique gaucha en tant que "bonne musique » ou comme " musique de qualité ». Ainsi, les organisateurs de la 'Californie' considéraient que ce contexte offrait une possibilité de " faire école » : favoriser la création de la musique qu'ils considéraient comme étant non-commerciale, qui était régie par des thèmes comme la grossièreté et le sexisme gaucho, dans le Rio Grande do Sul.

Avec les festivals de musique, le mouvement nativiste introduisit de nouvelles variantes dans une vieille dispute concernant la représentation légitime de l'identité de la musique régionale du Rio Grande do Sul. Ainsi, ils " revitalisèrent " (d'après Lucas) des mouvements régionalistes antérieurs et ont donné une impulsion nouvelle à la production musicale du Rio Grande do Sul. Les valeurs ajoutées à ces pratiques - surtout les pratiques musicales - se sont heurtées à de nombreux préceptes du Mouvement traditionaliste gaucho, ce qui évoque une rupture. Toutefois, cette rupture n'est qu'apparente, puisque l'analyse détaillée fait apparaître que la valeur du " vrai " gaucho est une préoccupation commune. Les deux mouvements s'efforcent l'un comme l'autre de distinguer le "vrai gaucho » de tout ce qui est perçu comme non authentique ; ils établissent en outre des partenariats pour la réalisation des festivals. Évidemment, ici, on se trouve dans un cadre très particulier dans lequel, une fois libérés des règles des Centres de tradition gaucha, les festivals nativistes créent leur propre loi. Ils établissent de nouveaux paradigmes, leur propre étiquette et leurs façons spécifiques de composer et d'écouter. Chaque festival établit son règlement pour exposer sa conception de la musique nativiste et définir les chansons qui peuvent concourir ${ }^{20}$.

19 "Em busca de algo novo " - publié le 27 septembre 1971. Porto Alegre, Folha da Tarde: 13.

20 Dans le cas du festival Califórnia, on crée des « lignes " caractérisées par les thématiques des compositions. Ainsi, une chanson pourrait être caractérisée comme « ligne campeira » - ligne identifiée à l'homme, au moyen, aux usages et aux costumes de la campagne du Rio Grande do Sul-, « ligne de manifestation rio-grandense » - liée à des aspects culturels et géographiques du Rio Grande do Sul 
Ainsi, il est possible d'établir un rapport entre l'émergence du sigle MPB et son identification comme genre musical, qui résulte des festivals des années 1960. Comme la MPB, la musique nativiste opère une sélection dans le répertoire des musiques régionales du Rio Grande do Sul et crée une forme de production musicale très spécifique. Cependant, il faut s'intéresser au concept de genre musical, tel qu'il s'entend ici, et à la façon dont une approche anthropologique de la relation entre les genres et les festivals musicaux peut contribuer au questionnement et à la réflexion sur les phénomènes du monde contemporain.

\section{Les festivals et les genres musicaux: une approche anthropologique}

Comme nous l'avons déjà montré, la MPB et la musique nativiste ont toutes deux produit des récits sur leurs inflexions musicales. En d'autres mots, alors que la première cristallise de nouvelles conceptions de ce qu'on appelle la musique populaire et brésilienne, la deuxième s'attache à définir ce qui devrait être compris comme étant une musique régionale et native du Rio Grande do Sul. Les événements festifs d'où ses genres sont issus sont des éléments clés dans ce processus, car leur dynamique prescrit des formes particulières de composition, d'exécution et d'audition. Évidemment, l'industrie du disque est aussi incontournable. Dans le cas du nativisme - comme l'a bien remarqué Nilda Jack (1998) - la phonographie est peut-être le secteur où l'impact du mouvement dans l'industrie culturelle gaucha est le plus évident. Cet impact est dû au fait que la plupart des festivals nativistes ont produit et produisent encore aujourd'hui des disques avec des compositions qui ont été jusqu'aux finales. La demande des festivals amène aussi l'émergence de nouvelles maisons de disques (Jacks 1998: 79-83).

D'après Holt (2008), le concept de genre musical se constitue à partir du dialogue entre de nombreux champs de connaissance de la musique. Cette association indique la façon dont la définition du genre, comme « une force structurante qui organise des pratiques culturelles et crée des contextes et des horizons pour la compréhension de la musique ", détermine l'avènement des musicologies. Ainsi, étant donné que la connaissance de la musique

\footnotetext{
différents de ceux abordés par la « ligne campeira » - et la « ligne de protection folklorique » - qui se base sur les autres lignes, mais qui considère l'universalité artistique du point de vue du traitement poétique musical (Oliven 1992: 117).
} 
dépend d'expériences culturelles et musicales spécifiques et que les différentes musicologies se sont développées à partir de musiques et de contextes historiques et culturels particuliers, ces musicologies se définissent l'une par rapport à l'autre et se disputent les espaces publics et le capital culturel. Il faut remarquer que cette dynamique est exactement la même pour les genres musicaux (Holt 2008: 42) ${ }^{21}$.

Cette question nous ramène à l'usage du concept de genre dans la perspective musicologique après les années 1980, comme le montrent Beard et Gloag (2005) dans le dictionnaire « Musicology: the key concepts ». Selon les auteurs, les musicologues commencent à utiliser le terme " genre " pour décrire les aspects " sociaux » et " extérieurs » d'une oeuvre, tandis que le mot style est appliqué à l'observation des caractéristiques « formelles » et " intérieures » de la musique. Le concept de genre est alors employé pour désigner les pratiques musicales en tant que fixes et « surgelées ». L'accent est mis sur ce qui est répété et vu comme étant cohérent " sans tenir compte du fait que quelques caractéristiques utilisées pour définir le genre, comme le style, la technique et la forme changent avec le temps " (Beard; Gloag 2005: 72). Même si les auteurs reconnaissent que, parfois, le genre et le style se confondent, on peut dire qu'à leur sens, il y a bien une distinction à opérer entre l' « objet » matériel (la musique) et le concept de genre musical (qui se rapporte aux signifiés imposés aux musiques par les « cultures musicales") (Beard; Gloag 2005: 72-73). Ainsi, par leur critique, Beard et Gloag semblent accepter cette dichotomie, surtout quand ils sont d'accord - dans une affirmation presque conclusive - avec l'idée du genre musical en tant que « convention sociale ». De plus, ils soulignent qu'il importe d'appliquer le concept de genre également aux études de la musique populaire, car pour eux, dans ce contexte, l'attachement aux genres est très important - à l'exemple des cultures «fan " - et les genres sont le produit du processus contemporain de

21 Pour l'auteur, normalement, le genre est défini par des processus de spécialisation musicale et sociale interconnectés. Ainsi, quand des individus se spécialisent dans une musique ou une tradition particulière, ils deviennent une partie d'une collectivité sociale qui produit une connaissance reconnue sur cette musique, car ils partagent des paradigmes et des valeurs (Holt 2008: 42). Par exemple, l'ethnomusicologie et les Popular Music Studies finissent par se constituer aux frontières de la musicologie occidentale, qui domine la plupart des départements universitaires de musique. Selon Holt, la musique sérieuse occidentale, surtout la musique allemande, a été le sujet privilégié de l'ancienne musicologie qui, jusqu'à nos jours, prédomine dans les recherches institutionnelles. Parallèlement, des stéréotypes de musiques " non occidentales " et " populaires " ont établi un décalage des études de musique populaire et d'ethnomusicologie par rapport à l'ethnomusicologie. 
mondialisation accélérée et d'innovations technologiques. Pour les auteurs, les transformations du monde contemporain et la croissante hybridité des pratiques musicales devraient être des incitations très importantes à mener une réflexion sur le concept de genre musical.

Cependant, nous croyons que la nécessité de réviser le concept de genre n'est pas due au fait que le monde contemporain produit une infinité de genres. De plus, nous ne sommes pas d'accord avec l'idée que ce sont les chercheurs du domaine des musiques populaires qui devraient mener cette réflexion parce qu'ils ont plus d'expérience avec le " populaire ", " l'extérieur " le matériau sonore - tandis que les musicologues étudient les aspects formels de la musique. Nous trouvons que la remise en question du concept procède, exactement, de la distinction opérée entre la " sphère des sons " et la " sphère sociale " - entités qui, d'une façon dangereuse, sont devenues des sujets d'analyse, comme le montre Strathern (2006).

Par ailleurs, l'approche des processus de territorialisation des genres musicaux et de la culture, d'une façon générale, est un aspect également important des études portant sur la musique populaire au XXème siècle, surtout en ce qui concerne les Amériques. Toutefois, dans les dernières décennies du XXème siècle et au début du XXIème, l'intérêt intellectuel à l'égard des déconstructions des concepts-clés augmente considérablement. Cet intérêt produit un déplacement des analyses vers des espaces considérés comme périphériques. Ce mouvement suscite des réflexions fondamentales pour la compréhension des musiques contemporaines et pour la révision du cadre d'analyse des histoires des musiques. D’après Carvalho et Segato (1994), la persistance de « l'hybridité des genres musicaux contemporains et leur autonomie par rapport aux territoires de culture ont des corolaires dans des époques passées et dans les sociétés dites traditionnelles. La transparence de leurs manifestations peut-être considérée comme leur particularité actuelle » (Carvalho; Segato 1994: 2). Cependant, la perspective déconstructiviste, qui est progressivement passée au centre de l'analyse, semble ne pas comprendre le rangement et le déplacement en tant que forces qui, au lieu de s'exclure, agissent ensemble.

Ainsi, des perspectives contemporaines, comme celles de Carvalho et Segato - et également de Holt - cherchent à admettre les difficultés de l'emploi du concept de genre musical et le danger de son rapport aux déterminismes ethniques et géographiques. Holt propose qu'au lieu de parler de 
genres musicaux, on considère que la musique se fasse " entre les genres " (music in-between genres) en élaborant une " poétique pour la compréhension de la musique entre les genres " (Holt 2008: 44). D'après l'auteur, cette poétique est construite d'une façon consciente autour d'un concept de genre décentralisé ; c'est-à-dire qui ne se base pas sur un modèle simpliste et délimité par la dichotomie entre centre et périphérie, mais qui est élaboré à partir de la considération des espaces entre les genres comme étant aussi importants que la notion de genre. Au contraire de réifier les divisions géographiques, par exemple, cette perspective essaie de se situer aux frontières, " aux lieux de passage et d'ambiguïté stylistique », comme le proposent Carvalho et Segato.

Si nous revenons au concept de genre selon le discours de Bakhtin pour penser la musique (1997) - appliqué aussi par Monson (1996), Piedade (2007; 2004), Domínguez (2009) et Menezes Bastos (1995b) -, nous voyons à quel point il importe de réviser le concept et son usage, non seulement en ce qui concerne les contextes de mondialisation et d'émergence des genres hybrides, mais aussi dans l'approche de la 'dynamicité' constituante de ces genres - dynamicité méprisée dans de nombreuses recherches. A partir de la lecture de Bakhtin, on peut dire que les genres de langage sont formés par trois éléments : le contenu thématique, le style verbal et la construction au niveau de la composition, des éléments qui forment toutes les énonciations. Au contraire de ce qu'on pourrait conclure, la stabilité des genres est assurée exactement par leur dynamicité. D’après Bakhtin, les trois éléments qui caractérisent toutes les énonciations sont marqués, surtout, par la spécificité d'une sphère de communication déterminée (Bakhtin 1997: p. 279). Par rapport à la musique, on pourrait dire que les genres présentent également des énonciations relativement stables pour ce qui est du style, de la construction de la composition et du contenu thématique. Ainsi, ils configurent un modèle de discours qui ne peut être reconnu que dans sa dynamicité et ses transformations dans une sphère de communication $^{22}$. Selon une perspective bakthinienne, une approche critique du genre musical devrait partir de l'idée selon laquelle il n'existe pas d'" explications sociales " pour tous les phénomènes d'ordre " sonore ».

Comme Menezes Bastos l'a démontré il y a déjà un certain temps, les phénomènes musicaux doivent être approchés en se démarquant à la fois « d’une

22 Fabbri (1981), de la même façon, considère que l'émergence des genres musicaux est due à une séquence d'événements musicaux dont le cours est gouverné et défini, mais d'une façon ouverte, par des règles fréquemment modifiées(p. 52). 
anthropologie sans musique et d'une musicologie sans homme ". L'idée de stabilité est complexifiée. En ce sens, nous croyons que l'analyse du contexte des festivals dérange quelques modèles anciens d'étude de la musique, puisqu'elle focalise les énonciations mises en action (dynamisées) dans une sphère de communication spécifique. Les festivals sont les scènes qui permettent la stabilité et en même temps la transformation des genres musicaux à travers l'interaction avec le groupe sonore, qui est toujours changeant ${ }^{23}$.

C'est dans le contexte festif que beaucoup de conceptions musicales des groupes sont exaltées et/ou établies par la performance musicale et l'effervescence du concours. D'après Napolitano (2010), ces « situations " d'écoute montrent qu'une réflexion sur la question de la réalisation sociale de la chanson est également nécessaire, c'est-à-dire, son appropriation et re-signification par l'audience dans un contexte spécifique (Napolitano 2010: 397). Ainsi, on pourrait se demander comment l'écoute individuelle d'un phonogramme dans l'espace privé se différencie de l'écoute collective d'une performance live dans un festival de musique? Quels sont les signifiés actionnés par cette situation?

D’après Lucas, la littérature anthropologique et sociologique, d'une façon générale, met l'accent sur les « inversions symboliques " des fêtes et des festivals. Dans le cas du Brésil, l'analyse des événements festifs privilégie la perspective de la construction d'un espace de critique, capable de condenser, illuminer et dénuder des situations clés dans la remise en question de l'état des choses du social. Pour Roberto DaMatta (1998), les festivités, principalement les fêtes populaires, mettent en scène le « drame " selon lequel le monde est inversé. En ce sens, au Brésil, les fêtes populaires, les commémorations civiques et les solennités religieuses forment un "système " très évident ou un " triangle rituel " ${ }^{24}$. Avec la combinaison de la tradition et de la modernité, le système des fêtes brésiliennes mène à l'inversion des rôles sociaux,

23 Le concept de groupe sonore a été développé par John Blacking (1995) et désigne « un groupe de personnes qui partage un langage musical, avec des conceptions sur la musique et ses usages " (Blacking 1995: 232). Ainsi, le groupe sonore englobe les musiciens, les chercheurs (académiques ou non) et aussi l'audience qui pense et parle de musique.

24 D’après DaMatta (1997), la notion de « triangle rituel » se réfère aux trois moments festifs les plus importants du Brésil et qui ont une portée nationale: la Semaine de la Patrie, la Semaine sainte et le Carnaval. D’après l'auteur, ces trois semaines festives constituent le « triangle rituel " puisque ce sont des festivités contrôlées par l'État, par l'église et par la société civile désorganisée. Ainsi, chaque moment festif extraordinaire correspond à un groupe ou à une catégorie sociale qui occupe une place définie - « son heure et son tour " - dans le cadre de la vie sociale nationale (DAMATTA, 1997: 53). 
ce qui est rendu possible au moins sur le plan symbolique ${ }^{25}$; il en résulte une " vision relationnelle ", en tant qu'alternative à une modernité, marquée par l'hégémonie de l'individualisme et par la notion bourgeoise d'égalité établie par la loi (DaMatta 1998: 76).

Comme l'a montré Maria Laura Viveiros de Castro Cavalcanti (2006) pour ce qui est des compétitions festives du carnaval brésilien, il faut analyser le caractère médiateur de ces festivités en tant que dispositif rituel d'articulation et d'expression des différences et comprendre le rituel en tant que processus rituel - notion proposée par Turner (1974). En ce sens, la compétition révèle l' « ambivalence intrinsèque de la réciprocité sociale: être en relation est aussi se confronter " (Cavalcanti 2006: 31). La rivalité suscitée par les compétitions des festivals est contrôlée par les règles qui sont établies et par une étiquette esthétique qui, bien que faisant l'objet d'une constante critique de la part des participants, est le moteur du moment paroxystique qui caractérise ces événements.

D’après Lucas, les festivals prennent une forme institutionnalisée dans les sociétés post-industrielles, où la participation atteint de vastes proportions. Pour Beverly Stoeltje (1992), ces constructions modernes mettent parfois en scène des intérêts idéologiques, commerciaux et politiques qui sont les fondements des sociétés qui les produisent. Ainsi, elles mènent, à certains moments privilégiés, à l'observation du dialogue entre différentes auto-interprétations des sociétés. A cet égard, quand on considère les genres musicaux en tant que système dynamique de discours (Menezes Bastos 2005a: 8), avec des frontières fluides et caractérisées par des disputes de significations menées par les différents groupes qui les constituent et qu'ils constituent (Domínguez 2009: 21), on peut réfléchir au rôle que jouent les fêtes et les festivals en tant qu'espaces où se formule la critique à l'égard de la formation de groupes sociaux, à partir des genres musicaux et vice-versa. Nous considérons cette question comme étant un point central en vue d'une approche anthropologique des genres musicaux. Elle révèle le besoin de réviser des visions dichotomiques qui séparent le contexte musical de la musique elle-même. Il faut étudier l'importance de ce rapport et les signifiés qu'il met en place, avant d'analyser la musique en tant que simple reflet privilégié 
d’identités sociales déjà établies ${ }^{26}$.

\section{Bibliographie}

AMARAL, Rita de Cássia. 1998. "A alternativa da festa à brasileira”. Sexta Feira, ano 2., n.2, pp. 108-115.

BAKHTin, Mikhail. 1997. Estética da Criação Verbal. São Paulo: Martins Fontes.

BARBOSA, Lívia. 2000. O Brasil não é para principiantes: carnavais, malandros e heróis, 20 anos depois. Rio de Janeiro: FGV.

CAVAlCANTI, Maria Laura Viveiros de Castro. 2006. Carnaval Carioca: dos bastidores ao desfile. Rio de Janeiro: Editora UFRJ, $3^{\text {a }}$ Ed.

CARVALHO, José Jorge de e SEGATO, Rita. 1994. "Sistemas abertos e territórios fechados: para uma nova compreensão das interfaces entre música e identidades sociais”. Série Antropologia 164, pp. 1-11.

DAMATTA, Roberto. 1998. "A mensagem das festas: reflexões em torno do sistema ritual e da identidade brasileira”. Sexta Feira, ano 2, n.2, pp.72-81.

DAMATTA, Roberto. 1997. Carnavais, malandros e heróis: uma sociologia do dilema brasileiro. Rio de Janeiro: Rocco.

DOMÍNGUEZ, Maria Eugenia. 2009. Suena El Río. Entre tangos, milongas, murgas e candombes: músicos e gêneros rio-platenses em Buenos Aires. Thèse, Université fédérale de Santa Catarina - Florianópolis.

FABBRI, Franco. 1982. "A theory of musical genres: two applications”. In: D. Horn; P. Tagg (eds.), Popular music perspectives. Gothenburg e Exeter: IASPM, pp. 52-81.

HOLT, Fabian. 2008. “A View from Popular Music Studies: Genre Issues”. In: Henry Stobart (Ed.), The New Ethno (musicologies). Lanhan, Maryland; Toronto; Plymouth, UK: The Scarecrow Press, Inc.

JACKS, Nilda. 1998. Mídia nativa: indústria cultural e cultura regional. Porto Alegre: Editora da UFRGS.

JACQUES, João Cezimbra. 1979. Assuntos do Rio Grande do Sul. Porto Alegre: União de Seguros Gerais.

LESSA, Barbosa e CÔRTES, Paixão. 1975. Danças e andanças da tradição gaúcha. Porto Alegre: Garatuja.

LUCAS, Maria Elizabeth da Silva. 1990. Gauchos on Stage: Regionalism, Social

26 Merci à Tatyana Jacques pour la traduction. 
Imagination and Tradition in the Festivals of Musica Nativa, Rio Grande do Sul, Brazil. Thèse, The University of Texas - Austin.

MANN, Henrique. 2002. Som do Sul: a história da música do Rio Grande do Sul no século XX. Porto Alegre: Tchê.

MARCON, Fernanda. 2009. Música de Festival: uma etnografia da produção de música nativista no festival Sapecada da Canção Nativa em Lages-SC. Mémoire de master, Universidade Federal de Santa Catarina- Florianópolis.

MARCON, Fernanda. 2010. "Música Nativista e Imaginários Gauchescos: sobre cantar opinando". Revista Música \& Cultura, n.5, pp. 1-9.

MELlo, Zuza Homem de. 2003. A era dos festivais: uma parábola. São Paulo: Editora 34.

MENEZES BASTOS, Rafael José de. 2005a. "Les Batutas, 1922: uma antropologia da noite parisiense.” Revista Brasileira de Ciências Sociais, vol.20, n. 58, pp.178-213.

MENEZES BASTOS, Rafael José de. 1999. "Músicas Latino-Americanas, Hoje: Musicalidade e Novas Fronteiras”. In: Música Popular en América Latina Atlas del II Congreso Latinoamericano IASPM. Santiago: FONDART, pp. 17-39.

MENEZES BASTOS, Rafael José de. 2009. "MPB, o Quê? Breve história antropológica de um nome, que virou sigla, que virou nome”. Antropologia em Primeira Mão, v.116, pp. 1-18.

MONSON, Ingrid T. 1996. Saying something: jazz improvisation and interaction. Chicago \& London: The University of Chicago Press.

NAPOLITANO, Marcos; WASSERMAN, Maria Clara. 200o. "Desde que o samba é samba: a questão das origens no debate historiográfico sobre a música popular brasileira”. Revista Brasileira de História, vol. 20, n.39, pp. 167-189.

NAPOLITANO, Marcos. 2007. A síncope das idéias: a questão da tradição na música popular brasileira. São Paulo: Fundação Perseu Abramo.

NAPOLITANO, Marcos. 2010. "MPB: a trilha sonora da abertura política (1975/1982)". Revista Estudos Avançados, 24 (69), pp: 389-402.

OLIVEIRA, Sílvio; VERONA, Valdir. 2006. Gêneros musicais campeiros no Rio Grande do Sul: ensaio dirigido ao violão. Porto Alegre: Nativismo.

OLIVEN, Ruben. 1992. A parte e o todo: a diversidade cultural do Brasil-Nação.

Petrópolis: Vozes.

ORTIZ, Renato. 2005. Cultura Brasileira e Identidade Nacional. São Paulo: 
Brasiliense.

PIEDADE, Acácio Tadeu de Camargo. 2007. "Expressão e sentido na música brasileira: retórica e análise musical”. Revista Eletrônica de Musicologia, vol. XI, pp. 1-15.

PIEDADE, Acácio Tadeu de Camargo. 2004. O canto do kawaká: música, cosmologia e filosofia entre os Wauja do Alto Xingu. Thèse, Université fédérale de Santa Catarina - Florianópolis.

QUEIROZ, Maria Isaura P. de. 1992. Carnaval Brasileiro: o vivido e o mito. São Paulo : Brasiliense.

SANTI, Álvaro. 2004. Do Partenon à Califórnia: o nativismo e suas origens. Porto Alegre: UFRGS.

STOELTJE, Beverly. 1992. "Festival”. In: Richard Bauman (Ed), Folklore, cultural performances and popular entertainments. New York: Oxford University Press.

STRATHERN, Marilyn. 2006. O gênero da dádiva. Campinas: Unicamp. TEIXEIRA, Sérgio Alves. 1988. Os recados das festas: representações e poder no Brasil. Rio de Janeiro: FUNARTE/Instituto Nacional do Folclore.

\section{Journaux}

“Em busca de algo novo”. Le 27 septembre 1971. Folha da Tarde. Porto Alegre: 13.

\section{Sites |internet}

O Toque - http://www.otoque.com.br/detalhes_entrevista_outras.asp?id_ cat=1. (consulté le 29 septembre 2010)

Blog Solano Ribeiro - http://solanoribeiro.blogspot.com/. (Accès à 26 septembre 2010)

Rede Histórica - http://historica.me/video/geraldo-vandre-quebrao\#ixzz1of7glBFW. (consulté le 27 septembre 2010) 


\section{À propos de l'auteur}

Fernanda Marcon prépare un doctorat dans le cadre du Programme d'études supérieures en anthropologie sociale de l'Université Fédérale de Santa Catarina et est chercheuse au MUSA - Centre de recherche sur l'art, la culture et la société en Amérique latine et les Caraïbes.

Adresse postale:

Luiz Thomaz Bondage Jacques, 179. Lagoa da Conceição, CEP: 88062-558, Florianópolis-SC.

E-mail: fersociais@yahoo.com.br 